\title{
Discovery of four super-soft X-ray sources in XMM-Newton observations of the Large Magellanic Cloud
}

\author{
C. Maitra and F. Haberl
}

\begin{abstract}
Max-Planck-Institut für extraterrestrische Physik, Gießenbachstraße 1, 85748 Garching, Germany e-mail: fwh@mpe.mpg.de
\end{abstract}

Received 5 September 2021 / Accepted 3 October 2021

\begin{abstract}
Context. Super-soft X-ray sources were established as a heterogeneous class of objects from observations of the Large Magellanic Cloud (LMC).

Aims. We have searched for new sources of this class in the X-ray images obtained from the XMM-Newton survey of the LMC and additional archival observations.

Methods. We first selected candidates by visual inspection of the image and screened out the artefacts that can mimic super-soft $\mathrm{X}$-ray sources as well as the bright foreground stars that create optical loading in the detectors. We obtained four new super-soft X-ray sources for which we performed detailed X-ray timing and spectral analyses and searched for possible optical counterparts to identify their nature. We also looked at archival ROSAT and Swift observations to investigate the long-term behaviour of the sources.

Results. XMMU J050452.0-683909 is identified as the central star of the planetary nebula SMPLMC 21 in the LMC. We suggest XMMU J051854.8-695601 and XMMU J050815.1-691832 as new soft intermediate polars based on the nature of their X-ray spectrum. Their estimated absorption-corrected luminosities and the blackbody radii indicate that they are located in our Galaxy, rather than the LMC. We discovered coherent pulsations of $497 \mathrm{~s}$ from XMMU J044626.6-692011, which indicates a magnetic cataclysmic variable nature of the source. The location of XMMU J044626.6-692011 in the LMC or our Galaxy is less clear. It could either be a white dwarf in the LMC with nuclear burning on its surface near the Eddington limit or another soft intermediate polar in our Galaxy. Conclusions. The discovery of new super-soft X-ray sources makes a significant contribution to the known population in our Galaxy. An observed higher density of sources in the direction of the Magellanic Clouds can likely be explained by the relatively low Galactic column density in their direction as well as a large number of existing observations sensitive at low X-ray energies.
\end{abstract}

Key words. novae, cataclysmic variables - white dwarfs - binaries: close - galaxies: individual: LMC - X-rays: stars

\section{Introduction}

The low Galactic foreground absorption in the direction of the Magellanic Clouds makes them ideal laboratories for the detection and investigation of super-soft X-ray sources (SSSs). In fact, the first SSSs were discovered in the Large Magellanic Cloud (LMC) using the Einstein observatory (Long et al. 1981) before further discoveries with ROSAT established them as a new, though heterogeneous, class of objects (see Greiner 1996; Kahabka \& Haberl 2006; Kahabka et al. 2008).

Super-soft X-ray sources are characterised by soft X-ray spectra with $k T \sim 15-80 \mathrm{eV}$ (e.g., Kahabka \& van den Heuvel 1997) and a wide range of luminosities. The most luminous $\left(\sim 10^{36}\right.$ to $\left.\sim 10^{38} \mathrm{erg} \mathrm{s}^{-1}\right)$ can be explained by stable nuclear burning white dwarfs (WDs), which accrete H-rich matter from a companion star (van den Heuvel et al. 1992). The size of the emission area derived from blackbody fits to their spectra is consistent with emission from the full WD surface. At least five of these close-binary SSSs are known in the LMC (Kahabka et al. 2008). White dwarfs as central stars of planetary nebulae (PNe) are known as SSSs in the LMC (SMP LMC 29; Kahabka et al. 2008) and the Small Magellanic Cloud (SMC; SMP SMC 22 and SMP SMC 25; Mereghetti et al. 2010).

A soft blackbody-like emission component is also observed from magnetic cataclysmic variables $(\mathrm{mCVs})$, albeit with significantly lower luminosities, below $10^{34} \mathrm{erg} \mathrm{s}^{-1}$. AM Her-type systems (polars; Cropper 1990) and DQ Her-type systems (soft intermediate polars; Haberl \& Motch 1995; Burwitz et al. 1996;
Anzolin et al. 2008) show pulsations in their X-ray flux that are caused by the rotation of the magnetised WD, which is synchronous with the orbital revolution in polars (typically longer than one hour). Typical WD spin periods of a few hundred seconds (Kuulkers et al. 2006) are found from intermediate polars (IPs) with $P_{\text {spin }} \sim 0.1 P_{\text {orb }}$ (Norton et al. 2004). Doubledegenerate binaries (i.e. systems that host two interacting WDs) can have orbital periods as short as a few minutes (Cropper et al. 1998; Esposito et al. 2014).

In this paper we report the discovery of new SSSs in XMMNewton observations of the LMC. In Sect. 2 we describe the $X M M-N e w t o n$ and other available archival X-ray observations as well as the analysis methods. Section 3 examines the OGLE data, which cover the X-ray positions. The results from our temporal and spectral analyses and our search for optical counterparts are presented in Sect. 4 and discussed in Sect. 5.

\section{X-ray observations and analysis}

\subsection{XMM-Newton}

In the course of the XMM-Newton surveys of the SMC (Haberl et al. 2012; Sturm et al. 2013) and the LMC (Maggi et al. 2016), we searched for new SSSs in the data obtained by the European Photon Imaging Cameras (EPIC), including pn- (Strüder et al. 2001) and MOS-type (Turner et al. 2001) charge-coupled device (CCD) detectors. Due to their soft X-ray spectra with the bulk of the emission below $\sim 1 \mathrm{keV}$, they appear as red sources in our 

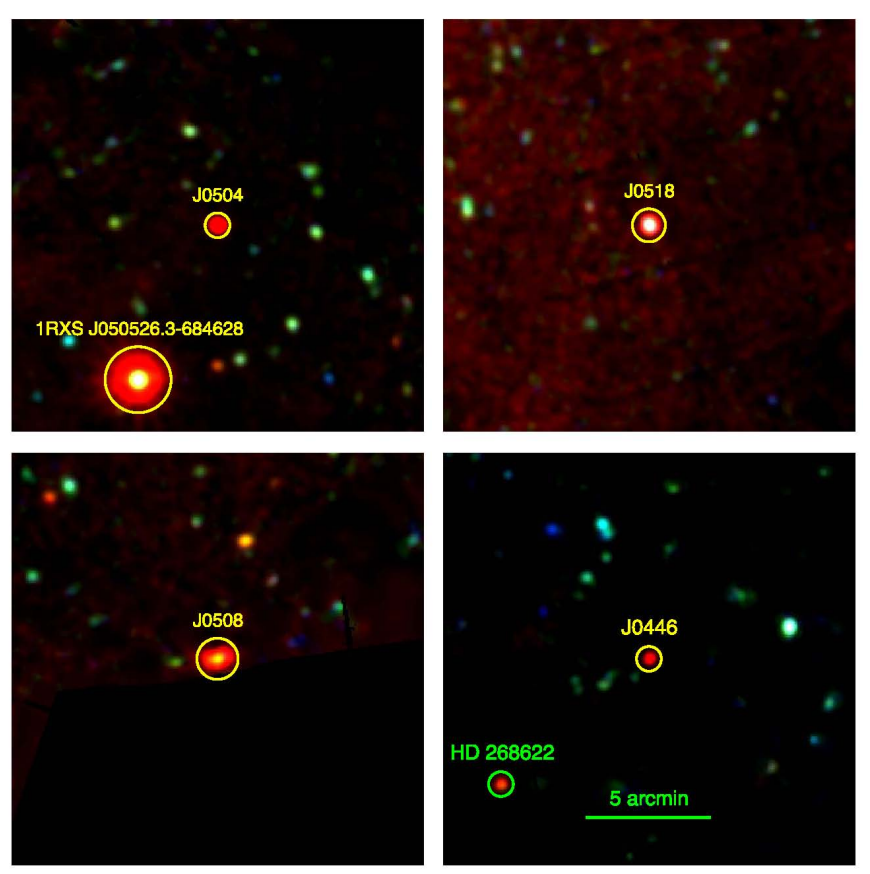

Fig. 1. Regions around the four new SSSs. The zoomed-in images are all on the same scale and extracted from the full RGB mosaic of the EPIC LMC observations. HD 268622 is a bright foreground star of spectral type G5 and $V=11.3 \mathrm{mag}$, which causes optical loading (see Sect. 2.1).

colour red-green-blue (RGB) images, which are composed of combined EPIC images in three energy bands (R: $0.2-1.0 \mathrm{keV}$, G: $1.0-2.0 \mathrm{keV}$, and B: $2.0-4.5 \mathrm{keV})$. The images were produced using the same pipeline as for the SMC as described in Sturm et al. (2013).

However, CCD detectors can also produce artefacts that mimic SSSs: (1) Hot CCD pixels or columns create events in low detector channels, and (2) optically bright stars produce optical loading due to the sensitivity of the CCDs to optical photons, which can release a sufficiently high number of electrons to create a signal above the low-energy threshold of the detector. Both of these types of false detections can be recognised because they are not subject to blurring due to the point spread function (PSF) of the telescope and can be identified in detector images (type 1 ), or they correlate with optically bright stars and produce large numbers of invalid event patterns (type 2).

We processed all EPIC observations in the directions of the Magellanic Clouds using the XMM-Newton Science Analysis Software (SAS) 19.0.0 ${ }^{1}$ package to create event lists using the most recent software and calibration files. Following Sturm et al. (2013), we produced the images and ran source detection. From a visual inspection of the RGB images of the LMC, we identified 22 new SSS candidates. In the majority of cases, we identified a bright foreground star ( $V$ magnitudes were between 4.8 and 11.4) in observations for which the thin optical blocking filter was used, which was insufficient to suppress the optical light. Only in one case did we find a hot pixel that was not masked out automatically by the software (ObsID 0690744401, pn-CCD =2, RAWX $=20$, RAWY =154). After this careful screening, we were left with four new SSS candidates. Colour images are presented in Fig. 1, which also shows an example of the appearance of a bright foreground star. A summary of the XMM-Newton observations in which they were found is given in Table 1.

https://www. cosmos.esa. int/web/xmm-newton/sas
We extracted the events to produce spectra and light curves from circular regions around the source positions and nearby blank-sky areas. Due to the higher sensitivity of the pn detectors compared to the MOS detectors at low energies, we used only pn data, except for the timing analysis of XMMU J044626.6-692011 (see Sect. 4.4). Single- and doublepixel events (PATTERN 0-4) were selected, excluding known bad CCD pixels and columns (FLAG 0). For the spectra, we removed times of increased flaring activity when the background was above a threshold of 8 counts ks${ }^{-1} \operatorname{arcmin}^{-2}(7.0-15.0 \mathrm{keV}$ band). EPIC spectra were re-binned to have at least one count per bin, and C-statistic was used. The SAS tasks arfgen and rmfgen were used to generate the corresponding detector response files. The X-ray spectra were analysed with the spectral fitting package XSPEC 12.11.0k ${ }^{2}$ (Arnaud 1996). Errors are specified for $90 \%$ confidence, unless otherwise stated.

We modelled the EPIC-pn spectra with blackbody emission attenuated by photo-electric absorption. For three of the sources, a weak, but significant, high-energy tail is seen in the spectra. Because the shape of this component is not well constrained (the power law and bremsstrahlung both yield acceptable fits), we used a bremsstrahlung model as bremsstrahlung is generally seen in the X-ray spectra of mCVs (see e.g., Mukai 2017). We fixed the temperature at $10 \mathrm{keV}$ and assumed that blackbody and bremsstrahlung components are attenuated by the same $N_{\mathrm{H}}$. First, we assumed the sources are located in the LMC and used two column densities along the line of sight. One accounts for the Galactic foreground with solar abundances according to Wilms et al. (2000) and was fixed at the value obtained from H I measurements (Dickey \& Lockman 1990) ${ }^{3}$, the other (free in the fit), with metal abundances set to 0.5 , reflects the absorption by the interstellar medium of the LMC (Rolleston et al. 2002) and local to the source. Second, if the source could be closer and located in the Milky Way, we used only one column density with solar abundance, allowing it as a free parameter in the spectral fit. Luminosities were corrected for absorption and calculated assuming a distance of $50 \mathrm{kpc}$ in the case of LMC and $1-5 \mathrm{kpc}$ in the case of our Galaxy.

\subsection{Swift}

We produced long-term light curves from archival data of the Swift X-ray telescope (XRT) in a soft energy band (0.3$1.0 \mathrm{keV}$ or $0.3-1.5 \mathrm{keV}$, depending on the spectrum) with time bins of 1 day, using the online tool of the UK Swift Science Data Centre ${ }^{4}$, which is described in Evans et al. (2007) and Evans et al. (2009). This time binning ensures that the data from the observations (also with different ObsIDs), which were performed shortly after one another, are merged. We set the minimum fractional exposure to 0.005 to ignore exposures that were too short to allow the sources to be detected. The list of available observations can also be obtained from the data centre ${ }^{5}$. Five archival observations cover the position of XMMUJ050452.0-683909(J0504 for short), three of which have sufficient XRT exposure to detect the source (February 2012, September 2016, and June 2020). The XRT $0.3-1.0 \mathrm{keV}$ count rates during these observations were $\left(1.4_{-0.9}^{+1.5}\right) \times 10^{-3} \operatorname{cts~s}^{-1},\left(2.5_{-1.6}^{+2.7}\right) \times 10^{-3} \mathrm{cts} \mathrm{s}^{-1}$, and $\left(0.62_{-0.59}^{+1.2}\right) \times$

\footnotetext{
2 Available at https://heasarc.gsfc.nasa.gov/xanadu/ xspec/

Extracted using NASA's HEASARC web interface https:// heasarc.gsfc.nasa.gov/cgi-bin/Tools/w3nh/w3nh.pl

4 https://www.swift.ac.uk/user_objects/

5 https://www.swift.ac.uk/swift_live/
} 
C. Maitra and F. Haberl: Super-soft X-ray sources in XMM-Newton observations of the LMC

Table 1. XMM-Newton observations of new SSSs.

\begin{tabular}{|c|c|c|c|c|c|c|c|c|}
\hline \multicolumn{2}{|c|}{ Source name } & \multirow{3}{*}{$\begin{array}{l}\text { Observation } \\
\text { ID }\end{array}$} & \multirow[t]{3}{*}{ Start time } & \multirow{3}{*}{$\begin{array}{l}\text { Exp. } \\
(\mathrm{ks})\end{array}$} & \multirow{3}{*}{$\begin{array}{c}\text { Off-axis } \\
\text { angle } \\
\left({ }^{\prime}\right)\end{array}$} & \multirow{2}{*}{\multicolumn{2}{|c|}{ RA }} & \multirow{3}{*}{$\begin{array}{l}\text { Err } \\
\left({ }^{\prime \prime}\right)\end{array}$} \\
\hline XMMU J... & short & & & & & & & \\
\hline & & & & & & $(\mathrm{h} \mathrm{m} \mathrm{s})$ & & \\
\hline \multirow{2}{*}{$050452.0-683909$} & $\mathrm{~J} 0504$ & & & 44 & 7. & 2 & -68 & 0.41 \\
\hline & & 06 & & & 13 & 39 & -6 & 0.64 \\
\hline $051854.8-695601$ & J0518 & 0690751801 & 2012-12-28 21:30 & 22.95 & 5.5 & 051854.82 & -695601.5 & 0.40 \\
\hline $050815.1-691832$ & $\mathrm{~J} 0508$ & 0690752001 & 2012-09-22 02:29 & 22.05 & 13.8 & 050815.14 & -691832.4 & 0.43 \\
\hline $044626.6-692011$ & J0446 & 0801990301 & 2017-09-28 21:50 & 21.54 & 5.1 & 044626.62 & -692011.9 & 0.48 \\
\hline
\end{tabular}

Notes. Parameters are given for the EPIC-pn instrument. Source coordinates were determined by applying bore-sight correction using background active galactic nuclei (following Maitra et al. 2019). The position error includes statistical and remaining systematic uncertainties (0.33"). Exposure times are given after correcting for dead times and the removal of intervals with high background.

Table 2. eROSITA observations of XMMU J051854.8-695601.

\begin{tabular}{lcc}
\hline \hline Observation & Start time & Exp. $(\mathrm{ks})$ \\
\hline 700182 & $2019-11-27$ 06:07:32.9 & 31.5 \\
700201 & $2019-12-0212: 45: 50.4$ & 11 \\
700202 & $2019-12-0402: 46: 43.5$ & 9 \\
\hline
\end{tabular}

$10^{-3}$ cts s$^{-1}$, respectively. Also, five observations cover the position of XMMU J051854.8-695601 (J0518), one of which with an XRT exposure of nearly $2 \mathrm{ks}$ (March 2013). The $0.3-1.5 \mathrm{keV}$ XRT count rate during this observation was $\left(4.4_{-1.6}^{+2.1}\right) \times 10^{-3} \mathrm{cts} \mathrm{s}^{-1}$. Numerous serendipitous observations of XMMU J050815.1-691832(J0508) exist in the Swift archive, which allowed us to investigate the long-term variability between November 2008 and June 2016. No Swift observations cover the position of XMMU J044626.6-692011 (J0446).

We also used the online analysis tool for the Swift ultra-violet optical telescope (UVOT) ${ }^{6}$ to search for optical-UV counterparts in the UVOT data. The analysis tool is based on the Swift/UVOT (Roming et al. 2005) software package and consists of the following steps: summing of all the exposure fractions, performing source detection using the uvotdetect task (detection threshold of 6 and quality filtering applied), and applying magnitude dereddening using the $E(B-V)$ value at the query position according to Schlafly \& Finkbeiner (2011).

\section{3. eROSITA}

J0518 was in the field of view of eROSITA, the soft X-ray instrument on the Spektrum-Roentgen-Gamma (SRG) mission (Predehl et al. 2021), during the 'CalPV phase' (Haberl et al. 2021). To analyse the CalPV data, we used the eROSITA Standard Analysis Software System (eSASS, version eSASSusers_201009; Brunner et al. 2021). The data reduction and spectral analysis were performed in a similar way as described by Maitra et al. (2021) and Carpano et al. (2021), where details can be found. The details of the CalPV observations are given in Table 2.

\section{OGLE observations}

The fields in the LMC were also observed by the Optical Gravitational Lensing Experiment (OGLE; Udalski et al.

\footnotetext{
${ }^{6}$ https://www.ssdc.asi.it/mmia/index.php?mission= swiftmastr
}
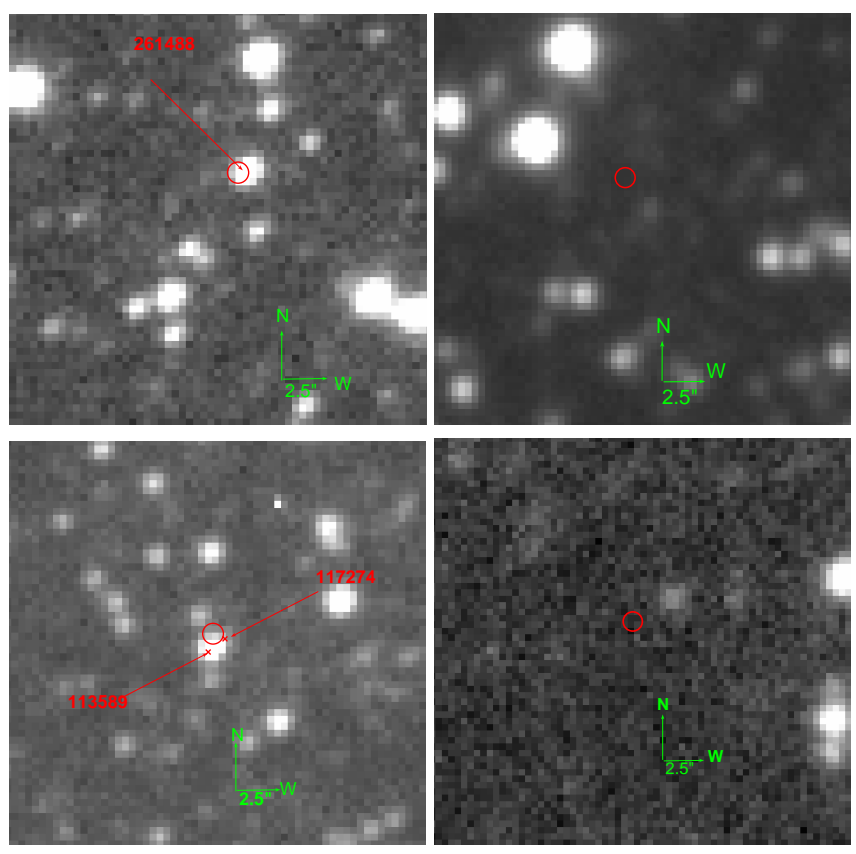

Fig. 2. Finding charts of the four new SSSs. In the zoomed-in $I$-band images from OGLE-II/III, the $1 \sigma$ error circles of the XMM-Newton positions and the OGLE source IDs are marked in red. The field identifications for J0504 (top left), J0518 (top right), J0508 (bottom left), and J0446 (bottom right) are OGLE-II LMC_SC14, OGLE-III LMC118.6, OGLE-II LMC_SC11, and OGLE-III LMC142.7, respectively.

1992), which provides more than 20 years of monitoring data (OGLE IV; Udalski et al. 2015). We looked at images taken in the $V$ and $I$ filter passbands, where the photometric magnitudes are calibrated to the standard VI system. For each source studied in this work, we searched for possible optical counterparts within the X-ray error circle given in Table 1 (see Fig. 2).

\section{Results}

\subsection{XMMUJ050452.0-683909}

The SSS candidate with the softest X-ray spectrum in our sample, J0504, was covered by two XMM-Newton observations (Table 1). It was detected in both observations in the lowest energy band $(0.2-0.5 \mathrm{keV})$ in pn and MOS images, which clearly excludes an instrumental origin. The EPIC-pn count rates in the soft band (corrected for vignetting) determined by the 

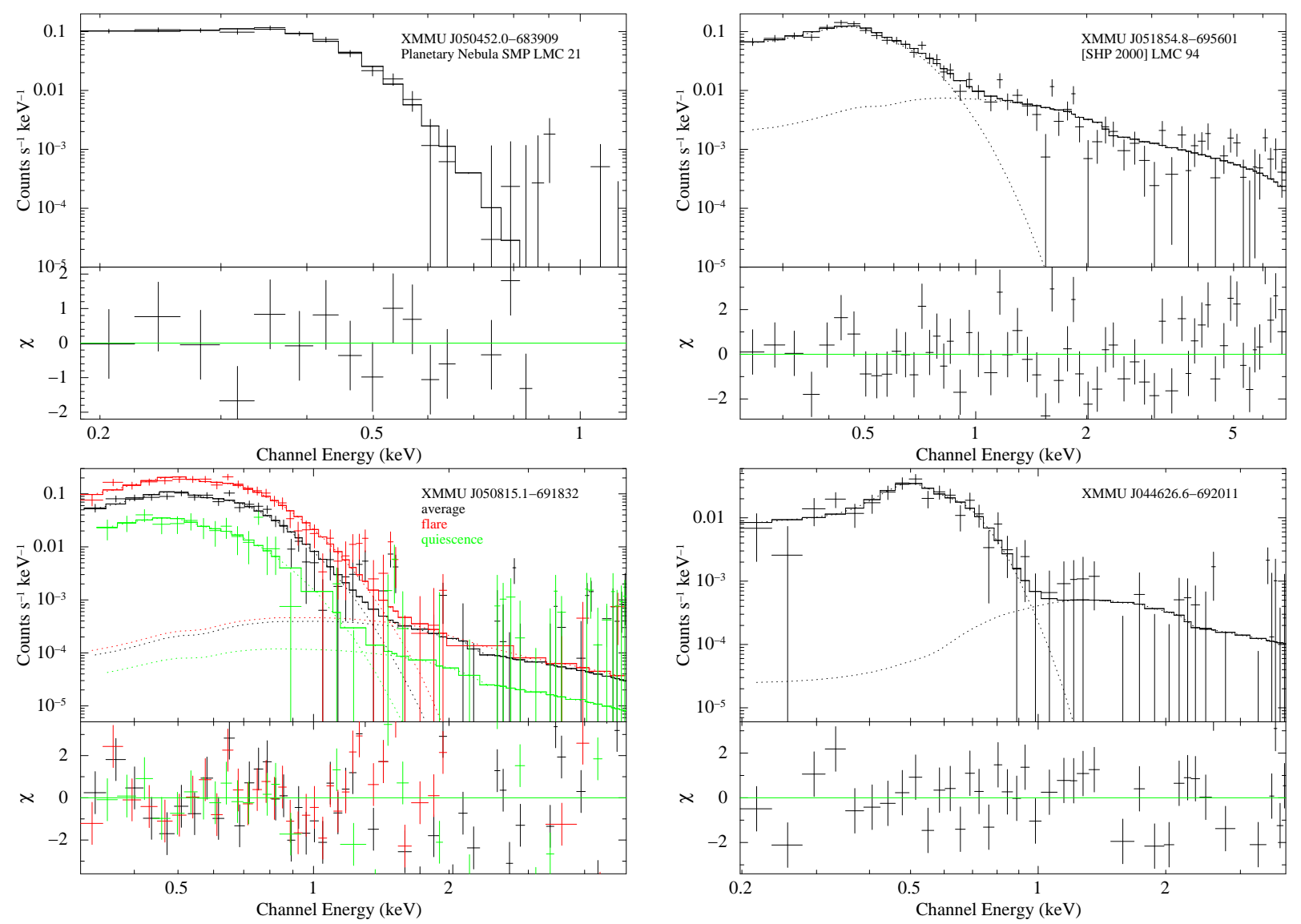

Fig. 3. EPIC-pn X-ray spectra of new SSSs observed in the XMM-Newton survey of the LMC. For J0508, the best-fit model was derived from the average spectrum, while the models for quiescent and flare emission were scaled by a constant factor, keeping all other parameters fixed at the values of the average spectrum.

source detection algorithm of $(4.11 \pm 0.36) \times 10^{-2} \mathrm{cts} \mathrm{s}^{-1}$ (February 2013) and 0.0455(14) $\mathrm{cts} \mathrm{s}^{-1}$ (October 2017) are consistent within their $(1 \sigma)$ errors. The source was observed at a very large off-axis angle in 2013. To avoid systematic uncertainties, we considered only the 2017 observation for the extraction of source products.

To search for an optical counterpart, we used the X-ray coordinates derived from the observation with a smaller off-axis angle. This position is right at the centre of the PN SMP LMC 21 (at $\mathrm{RA}=05^{\mathrm{h}} 04^{\mathrm{m}} 51^{\mathrm{s}} .99$ and $\mathrm{Dec}=-68^{\circ} 39^{\prime} 09^{\prime \prime} \cdot 7$ with angular extent of $0.23^{\prime}$; Reid \& Parker 2006, catalogue entry 1797). This is the second association of an SSS with a PN in the LMC after XMMU J050803.1-684017 = SMP LMC 29 (Kahabka et al. 2008), while two are detected in the SMC (Mereghetti et al. 2010). The OGLE counterpart of this object (LMC_SC14 StarID: 261488 ) is at $\mathrm{RA}=05^{\mathrm{h}} 04^{\mathrm{m}} 52^{\mathrm{s}} \mathrm{s} 05$ and $\mathrm{Dec}=-68^{\circ} 39^{\prime} 09^{\prime \prime} \cdot 9$ with $I=16.9 \mathrm{mag}$ (Fig. 2). The OGLE-II $I$-band light curve of the star does not exhibit significant variability.

The EPIC-pn spectrum is well represented by an absorbed blackbody component (Fig. 3 and Table 3). The inferred size of the emission area is consistent with emission from the full surface of a WD (see Table 3). The source intrinsic luminosity of $1.7 \times 10^{37} \mathrm{erg} \mathrm{s}^{-1}$ is very similar to that of SMP SMC 22 (Mereghetti et al. 2010) and consistent with being constant between the two XMM-Newton observations, which were taken almost 4.7 years apart. On the other hand, the source was not detected in ROSAT pointed observations. Using the best-fit model parameters derived from the EPICpn spectrum (obtained from the observation in 2017) and the detector response of the ROSAT position sensitve proportional counter (PSPC), a count rate of $3.5 \times 10^{-3} \mathrm{cts} \mathrm{s}^{-1}$ is expected. With such a count rate, the source should have been detected in two ROSAT/PSPC observations: 500037p (April 1992, 6826 s exposure) and 500258p (October-December 1993, 12715 s) with $\sim 20$ cts (taking into account vignetting), which is a factor of about three higher than the detection limit. In other words, J0504 was at least a factor of three fainter during the ROSAT observations. During Swift observations (2012, 2016, and 2020), J0504 was detected with $0.3-1.0 \mathrm{keV}$ count rates between $0.6 \times 10^{-3} \mathrm{cts} \mathrm{s}^{-1}$ and $2.5 \times 10^{-3} \mathrm{cts} \mathrm{s}^{-1}$. Assuming the EPIC-pn best-fit spectral parameters, an XRT count rate of $\sim 2.1 \times 10^{-3} \mathrm{cts} \mathrm{s}^{-1}$ is expected, consistent with the observations.

\subsection{XMMU J051854.8-695601}

J0518 was detected in the EPIC images of observation 0690751801 (Table 1) with an EPIC-pn count rate $(0.2-0.5 \mathrm{keV}$, corrected for vignetting) of $(4.29 \pm 0.20) \times 10^{-2} \mathrm{cts} \mathrm{s}^{-1}$. No optical counterpart was found for the object in the OGLE database (Fig. 2) nor in the Gaia catalogues within the X-ray error circle. A star with $V \sim 21 \mathrm{mag}$ was, however, found within the error circle from the Magellanic Clouds Photometric Survey (MCPS) catalogue of Zaritsky et al. (2004). No source was detected at the position of J0518 from the Swift/UVOT data. Using the longest UVOT exposure that covers J0518 (ObsID 00045502001, exposure $1014 \mathrm{~s}$, filter uvm2) and the sources detected in the field, a lower limit of $18.5 \mathrm{mag}$ was obtained. 
Table 3. Spectral fit results.

\begin{tabular}{|c|c|c|c|c|c|c|c|c|c|}
\hline $\begin{array}{l}\text { Source } \\
\text { name }^{(a)}\end{array}$ & $\begin{array}{c}\text { Observation } \\
\text { ID } \\
\end{array}$ & $\begin{array}{l}k T_{\mathrm{bb}} \\
(\mathrm{eV}) \\
\end{array}$ & $\begin{array}{c}N_{\mathrm{H}}^{\mathrm{Gal}} \\
\left(10^{21} \mathrm{~cm}^{-2}\right) \\
\end{array}$ & $\begin{array}{c}N_{\mathrm{H}}^{\mathrm{LMC}} \\
\left(10^{21} \mathrm{~cm}^{-2}\right) \\
\end{array}$ & $\begin{array}{l}\text { C-stat } \\
\text { /d.o.f. }\end{array}$ & d.o.f. & $\begin{array}{c}F_{\text {observed }}(c) \\
\left(\mathrm{erg} \mathrm{cm}^{-2} \mathrm{~s}^{-1}\right)\end{array}$ & $\begin{array}{c}L^{(d)} \\
\left(\mathrm{erg} \mathrm{s}^{-1}\right) \\
\end{array}$ & $\begin{array}{l}R_{\mathrm{BB}} \\
(\mathrm{km}) \\
\end{array}$ \\
\hline J0504 & 0803460101 & $31 \pm 4$ & 0.64 & $1.2_{-0.4}^{+0.5}$ & 0.66 & 19 & $7.6 \pm 0.4 \times 10^{-14}$ & $1.7 \times 10^{37}$ & $16000_{-9600}^{+40600}$ \\
\hline \multirow[t]{2}{*}{ J0518 } & 0690751801 & $78 \pm 8$ & 0.67 & $0.9_{-0.5}^{+0.6}$ & 1.2 & 94 & $1.0 \pm 0.1 \times 10^{-13}$ & $2.1 \times 10^{35}$ & $220_{-80}^{+1400}$ \\
\hline & eROSITA & $88 \pm 4$ & 0.67 & $0.09_{-0.09}^{+0.16}$ & 1.1 & 106 & $1.7 \pm 0.2 \times 10^{-13}$ & $2.0 \times 10^{35}$ & $135_{-20}^{+28}$ \\
\hline $\mathrm{J} 0508^{(b)}$ & 0690752001 & $86 \pm 9$ & 0.74 & $1.9_{-0.8}^{+0.09}$ & 1.7 & 54 & $1.7 \pm 0.1 \times 10^{-13}$ & $5.7 \times 10^{35}$ & $290_{-120}^{+280}$ \\
\hline J0446 & 0801990301 & $49 \pm 14$ & 0.72 & $6.4_{-3.2}^{+5.6}$ & 0.94 & 40 & $2.0 \pm 0.3 \times 10^{-14}$ & $7 \times 10^{36}$ & $3400_{-3000}^{+9600}$ \\
\hline \multirow[t]{2}{*}{ J0518 } & 0690751801 & $78 \pm 7$ & $1.6_{-0.4}^{+0.5}$ & - & 1.2 & 94 & $1.0 \times 10^{-13}$ & $9.0 \times 10^{31}$ & $4.5_{-1.6}^{+2.9}$ \\
\hline & eROSITA & $88 \pm 4$ & $0.75_{-0.13}^{+0.15}$ & - & 1.1 & 106 & $1.7 \times 10^{-13}$ & $6.0 \times 10^{31}$ & $2.7_{-0.5}^{+0.6}$ \\
\hline $\mathrm{J} 0508^{(b)}$ & 0690752001 & $87 \pm 9$ & $2.5_{-0.8}^{+1.0}$ & - & 1.7 & 54 & $1.7 \times 10^{-13}$ & $2.4 \times 10^{32}$ & $6.1_{-2.8}^{+6.7}$ \\
\hline J0446 & 0801990301 & $50 \pm 14$ & $7.1_{-3.3}^{+6.5}$ & - & 0.96 & 40 & $2.0 \times 10^{-14}$ & $5.0 \times 10^{33}$ & $87_{-78}^{+265}$ \\
\hline
\end{tabular}

Notes. Best-fit parameters using a model with absorbed blackbody and bremsstrahlung emission. The bremsstrahlung component is not well constrained, and the temperature was fixed at $10 \mathrm{keV}$ (see Sect. 2.1). The C-stat values include the full model. Errors indicate $90 \%$ confidence ranges. ${ }^{(a)}$ For full source names, see Table $1 .{ }^{(b)}$ Spectral parameters derived from the average spectrum. ${ }^{(c)}$ Fluxes are provided for the $0.1-2.4 \mathrm{keV}$ band to allow comparison with values published for SSSs based on ROSAT observations. ${ }^{(d)}$ Source luminosities $(0.1-2.4 \mathrm{keV})$ corrected for absorption, assuming a distance of $50 \mathrm{kpc}$ (Pietrzyński et al. 2013) in the upper part of the table. The Galactic foreground column density was taken from Dickey \& Lockman (1990). For the model in the lower part of the table, only a Galactic absorption component (with free column density in the fit) and a distance of $1 \mathrm{kpc}$ were assumed. A note of caution regarding absorption-corrected X-ray luminosities in soft energy bands: Column densities with large errors lead to a large uncertainty on the luminosity, which is in particular the case for J0446.

Due to the relatively small off-axis angle of the source and the high detection significance, the data were suitable for a detailed analysis. The EPIC-pn spectrum can be fitted by a blackbody and an additional bremsstrahlung component to account for a significant hard tail in the spectrum. Figure 3 shows the best-fit EPIC-pn spectrum, and the best-fit spectral parameters are tabulated in Table 3. The total $0.1-8.0 \mathrm{keV}$ absorption-corrected luminosity at the distance of the LMC is $2.13 \times 10^{35} \mathrm{erg} \mathrm{s}^{-1}$ with the bremsstrahlung component accounting for $11 \%$, demonstrating the dominance of the soft component in the spectrum. Although the blackbody luminosity depends somewhat on the temperature and absorption derived from the spectral fit, the luminosity is well below the range in which $\mathrm{H}$ burning is expected to be stable (Wolf et al. 2013). In addition, the radius of the emitting area of $220 \mathrm{~km}$ would be much smaller than the size of a WD. On the other hand, the X-ray luminosity would be too high to be explained by an $\mathrm{mCV}$ in the LMC. If we assume the source to be located in the Milky Way, the luminosity and radius of the emitting area are correspondingly smaller (e.g., for distances of 1-5 kpc: $L_{\mathrm{bol}}=9 \times 10^{31} \mathrm{erg} \mathrm{s}^{-1}$ to $2 \times 10^{33} \mathrm{erg} \mathrm{s}^{-1}$ and $R_{\mathrm{BB}}=4-20 \mathrm{~km}$; see also Table 3 ).

J0518 was observed during the CalPV phase of eROSITA. Data from the different telescope modules TM1, 2, 3, 4, and 6 (which are equipped with cameras with on-chip optical blocking filter) and from the three observations were added. The combined spectrum is well represented by the same models as in the case of XMM-Newton EPIC-pn; the best-fit parameters are listed in Table 3.

The eROSITA spectrum with the best-fit two-absorption component model is presented in Fig. 4. While the temperature and emission radius derived from the eROSITA spectrum are consistent with those measured from EPIC-pn, the column densities are significantly lower during the eROSITA CalPV phase in November 2019, almost seven years after the XMM-Newton observation. The higher absorption in 2012 results in a lower observed flux, but the absorption-corrected intrinsic luminosities during the two observations are similar, with a slightly lower value during the 2019 observations.

The source was detected previously in a ROSAThighresolution imager (HRI) observation (RH601040 in December

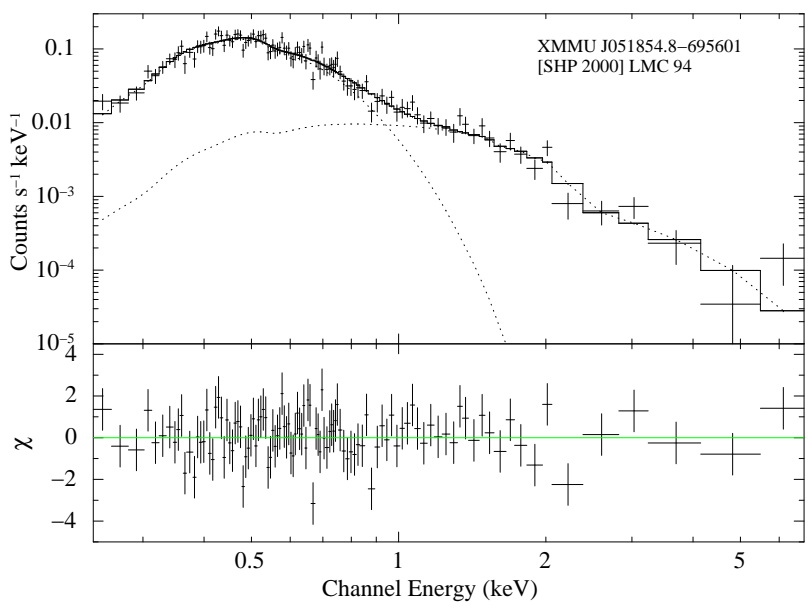

Fig. 4. Combined eROSITA X-ray spectrum of XMMU J051854.8695601 observed in the CalPV phase together with the best-fit model. The model consists of soft blackbody and hard bremsstrahlung components, which are indicated by dotted lines.

1997-January 1998) and is listed in the catalogue of Sasaki et al. (2000) as source 94 ([SHP2000] LMC 94) with a count rate of $(2.6 \pm 0.4) \times 10^{-3} \mathrm{cts} \mathrm{s}^{-1}$. Assuming the current spectral model derived from EPIC-pn, the expected HRI count rate is $\sim 2.2 \times 10^{-3} \mathrm{cts} \mathrm{s}^{-1}$, consistent with the ROSAT measurement. Similarly, the flux of $1.0 \times 10^{-13} \mathrm{erg} \mathrm{cm}^{-2} \mathrm{~s}^{-1}$ observed with EPIC-pn (in September 2012) corresponds to a Swift/XRT 0.3$1.5 \mathrm{keV}$ count rate of $\sim 4.1 \times 10^{-3} \mathrm{cts} \mathrm{s}^{-1}$, consistent with the detection in March 2013.

\subsection{XMMU J050815.1-691832}

J0508 was detected in the EPIC images of observation 0690752001 (Table 1) at an EPIC-pn count rate $(0.2-0.5 \mathrm{keV}$, corrected for vignetting) of $(6.25 \pm 0.30) \times 10^{-2} \mathrm{cts} \mathrm{s}^{-1}$. Although there was no OGLE counterpart within the X-ray error circle, the nearest counterpart, LMC_SC11 StarID: 117274, lies at 0.75" (just outside the error circle) with $I=20.2 \mathrm{mag}$ (Fig. 2). Due to 


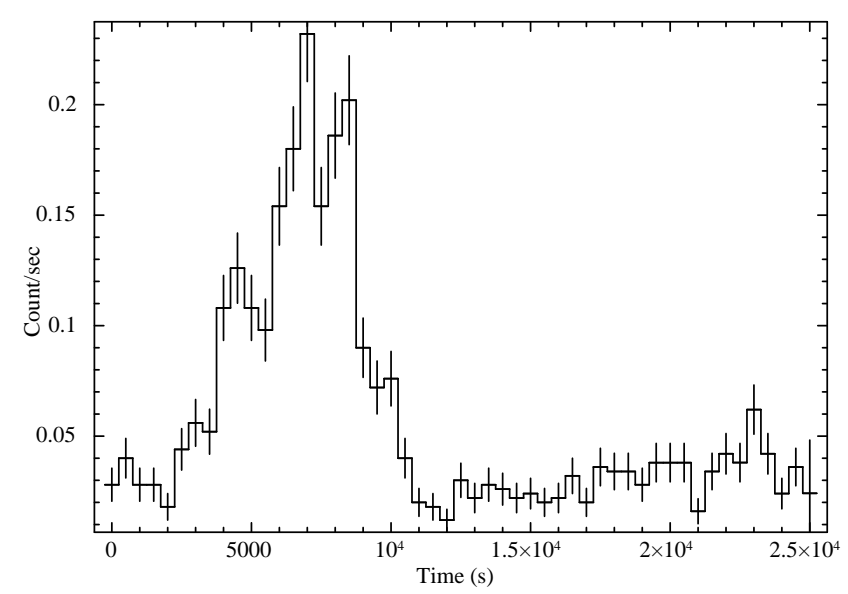

Fig. 5. EPIC-pn light curve of XMMU J050815.1-691832 (observation 0690752001 ) binned at $500 \mathrm{~s}$ in the $0.2-2 \mathrm{keV}$ band. Time zero corresponds to 22 September 2012 at 02:29:24 UTC.

the detection of the source near the edge of the field of view, the position may be uncertain, and we ascertain this as the plausible optical counterpart. The OGLE-II I-band light curve of the star does not exhibit variability, although this cannot be ruled out due to large uncertainties. No source was detected at the position of J0508 from the Swift/UVOT data. Using the longest UVOT exposure that covers J0518 (Obsid 00030348002, exposure $2049 \mathrm{~s}$, filter $w 1$ ) and the sources detected in the field, a lower limit of $19.1 \mathrm{mag}$ was obtained.

Although the source was detected at a large off-axis angle, its relatively bright nature allowed us to perform timing and spectral analyses of the EPIC-pn data. The EPIC-pn light curve of J0508 in the $0.2-2 \mathrm{keV}$ band shows flaring activity shortly after the beginning of the observation, which lasted for $\sim 8.2 \mathrm{ks}$ (Fig. 5).

The EPIC-pn spectrum can be described by blackbody emission with $k T=86 \mathrm{eV}$ and a weak bremsstrahlung component. The best-fit spectral parameters are summarised in Table 3 . The blackbody temperature of J0508 is similar to that of J0518, but significantly higher than that of J0504 and probably also J0446. The total unabsorbed luminosity at the distance of LMC is $6 \times 10^{35} \mathrm{erg} \mathrm{s}^{-1}$ with the bremsstrahlung component accounting for only $2 \%$ of the total $0.1-8 \mathrm{keV}$ emission, demonstrating again the dominance of the soft component. As in J0518, the luminosity is well below the range for stable $\mathrm{H}$ burning, and the radius of the emitting area of $\sim 290 \mathrm{~km}$ would be much smaller than the size of a WD. The inferred size of the emission region is indicative of hot spots on the WD surface and suggests an IP nature of the source. However, the X-ray luminosity would again be too high to be explained by an LMC membership of the source. If we assume a source location in the Milky Way, the luminosity and radius of the emitting area are correspondingly smaller (e.g., for distances of $1-5 \mathrm{kpc}: L_{\mathrm{bol}}=2 \times 10^{32} \mathrm{erg} \mathrm{s}^{-1}-5 \times 10^{33} \mathrm{erg} \mathrm{s}^{-1}$ and $R_{\mathrm{BB}}=6-30 \mathrm{~km}$; Table 3 ).

We also extracted spectra, as shown in Fig. 3, from the 'flaring' and 'quiescent' intervals, guided by the light curve in Fig. 5. The spectrum of the entire observation and the spectra from the flaring and quiescent intervals were simulatenously fitted. We left the blackbody temperature, the $N_{\mathrm{H}}^{\mathrm{LMC}}$, and a normalisation constant (to account for an overall change in flux) free. Due to the insufficient statistical quality of the spectra, no change in the spectral parameters, except the normalisation, could be detected within uncertainties. The peak luminosity dur-

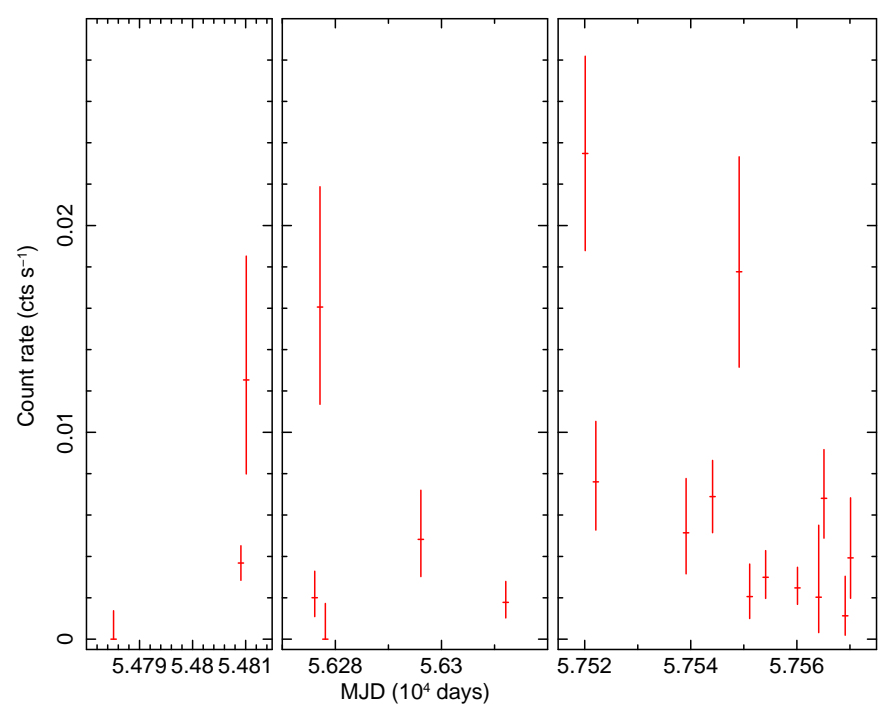

Fig. 6. Long-term X-ray light curve of XMMU J050815.1-691832 in the $0.3-1.5 \mathrm{keV}$ band with one-day binning obtained from archival Swift observations. The source was mainly observed during three epochs: November-December 2008, December 2012-January 2013, and May-June 2016.

ing the flaring interval was a factor of $\sim 10$ higher than during quiescence. Figure 3 shows the best-fit EPIC-pn spectra during the entire observation as well as during the flaring and quiescence intervals.

J0508 is consistent in position with 2RXS J050813.5691831, which was detected in the ROSAT all-sky survey (Boller et al. 2016) with a PSPC count rate of $1.2 \times 10^{-2} \mathrm{cts} \mathrm{s}^{-1}$, and the source is also listed in the ROSAT/HRI catalogue of Sasaki et al. (2000) with a count rate of $(4.9 \pm 0.7) \times 10^{-3}$ cts s$^{-1}$ ([SHP2000] LMC 38). Using the best-fit model parameters derived from the EPIC-pn spectrum and the ROSAT/PSPC and HRI detector responses, the predicted count rates are $\sim 1.2 \times 10^{-2} \mathrm{cts} \mathrm{s}^{-1}$ and $\sim 4.5 \times 10^{-3} \mathrm{cts} \mathrm{s}^{-1}$, respectively. This indicates that the source was at a consistent brightness level during the ROSAT all-sky survey (1990), the pointed ROSAT/HRI (RH601036 1997/1998), and the XMM-Newton (2012) observation.

The soft X-ray light curve of J0508 extracted from archival Swift data (Fig. 6) suggests some brightening during May-June 2016. Based on the EPIC-pn best-fit spectral model parameters, the observed flux of $1.7 \times 10^{-13} \mathrm{erg} \mathrm{cm}^{-2} \mathrm{~s}^{-1}$ corresponds to a Swift/XRT count rate of $\sim 7.4 \times 10^{-3} \mathrm{cts} \mathrm{s}^{-1}(0.3-1.5 \mathrm{keV})$. In June 2016, the source flux reached a maximum of about a factor of three higher, while the average count rate from the full Swift light curve of $(3.1 \pm 0.4) \times 10^{-3} \mathrm{cts} \mathrm{s}^{-1}$ was at about half the flux level during the ROSAT and XMM-Newton observations.

\subsection{XMMU J044626.6-692011}

J0446 was detected in the EPIC images observation 0801990301 (Table 1) at an EPIC-pn count rate $(0.2-0.5 \mathrm{keV}$, corrected for vignetting) of $(8.9 \pm 0.8) \times 10^{-3} \mathrm{cts} \mathrm{s}^{-1}$. No optical counterpart was found for the object in the OGLE database (Fig. 2) nor in the Gaia catalogues within the X-ray error circle.

The source was detected at a relatively small off-axis angle, therefore allowing detailed timing and spectral analyses of the EPIC-pn data. We first analysed the EPIC-pn light curve in 


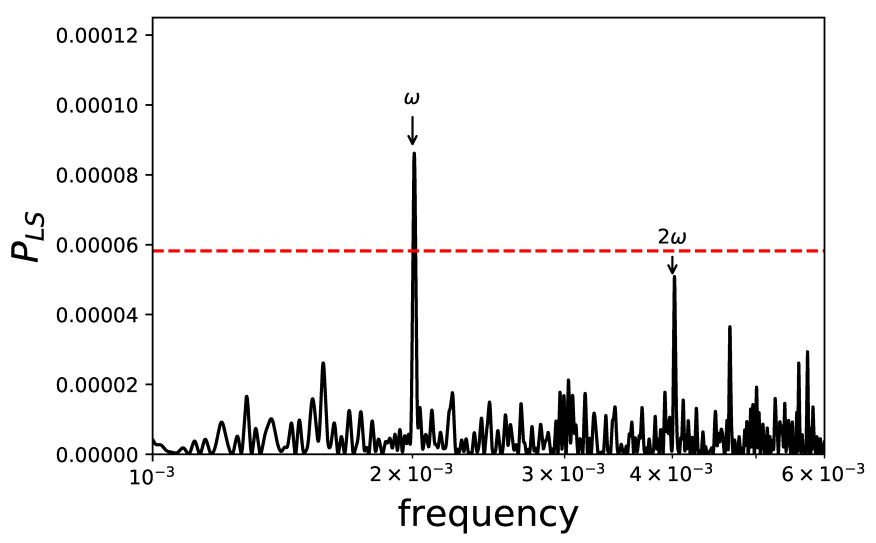

Fig. 7. Lomb-Scargle periodogram of the combined EPIC light curve of XMMU J044626.6-692011 (0.2-2 keV). The dashed red line marks the $3 \sigma$ confidence level.

the energy range $0.2-2 \mathrm{keV}$ using a Lomb-Scargle periodogram analysis (Lomb 1976; Scargle 1982). A period was found at $\sim 496$ s along with the first harmonic. The signal was somewhat enhanced when using the combined EPIC (pn+M1+M2) light curve, and the periodogram is shown in Fig. 7. The pulse period and its associated $1 \sigma$ error were determined to be $497.4 \pm 1.6 \mathrm{~s}$. The XMM-Newton EPIC-pn light curve in the range of $0.2-$ $2 \mathrm{keV}$, folded with the best-obtained period, is shown in Fig. 8. The period is in the typical range observed from IPs and likely indicates the spin period of the WD.

The EPIC-pn spectrum is well represented by an absorbed blackbody component, with an indication for a weak hard tail (Fig. 3). The best-fit spectral parameters are listed in Table 3. Because of very large uncertainties in temperature and absorption, the luminosity of the blackbody component cannot be reliably determined. Assuming the formally best-fit parameters, the contribution of the bremsstrahlung emission to the total luminosity is completely negligible and could also be caused by a background-subtraction problem. Assuming J0446 is located at LMC distance, the inferred size of the emission area is consistent with emission from the full surface of a WD. The measured LMC $N_{\mathrm{H}}=6.4 \times 10^{21} \mathrm{~cm}^{-2}$ is the highest of the sources studied in this work and leads to an unabsorbed luminosity of $7 \times 10^{36} \mathrm{erg} \mathrm{s}^{-1}$. We caution that this value is strongly dependent on the actual absorption along the line of sight to this source. If we assume a source location in the Milky Way at a distance of $1 \mathrm{kpc}$, the luminosity and radius of the emitting area are $L_{\text {bol }}=5 \times 10^{33} \mathrm{erg} \mathrm{s}^{-1}$ and $R_{\mathrm{BB}}=87 \mathrm{~km}$ (Table 3 ).

J0446 is located at the western rim of the LMC and was only covered by a short $(9 \mathrm{ks})$ ROSAT/HRI observation with the source at a large off-axis angle of $\sim 17^{\prime}$, which does not yield a stringent upper limit for the flux. No pointed ROSAT observations with the more sensitive PSPC detector nor Swift observations were performed at that location.

\section{Discussion}

From a systematic search for new SSSs in the XMM-Newton data of the Magellanic Clouds, we report the discovery of four sources in the direction of the LMC. We also used OGLE data to search for their possible optical counterparts and used archival ROSAT and Swift observations to investigate their longterm behaviour. One of the sources, XMMU J051854.8-695601, was in the field of view of eROSITA during the CalPV phase and was studied in this work.

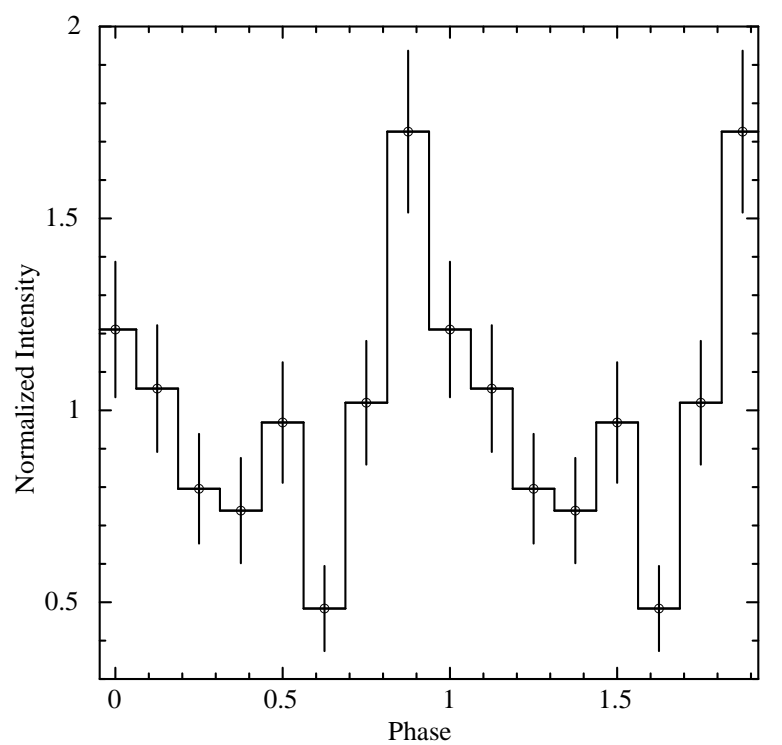

Fig. 8. EPIC-pn light curve of XMMU J044626.6-692011 in the 0.2$2 \mathrm{keV}$ band folded at the best-fit spin period.

\subsection{XMMU J050452.0-683909 = SMP LMC 21}

J0504 is identified as the central star of the PN SMP LMC 21 in the LMC. SMPLMC 21 is a high excitation PN (Morgan 1984) that exhibits a quadrupolar morphology of $1.15^{\prime \prime}$ extent (Stanghellini et al. 1999). The chemical composition from optical observations suggests a Type I PN hosting a relatively massive progenitor from the asymptotic giant branch (Stanghellini et al. 2007, and references therein). Using archival observations from 2009, Mereghetti et al. (2010) derived an upper limit of the unabsorbed X-ray luminosity of $3 \times 10^{35} \mathrm{erg} \mathrm{s}^{-1}$ (see Table 5 in their paper). In this work we detect the source with high confidence, and our results indicate a much higher $\mathrm{X}$-ray luminosity. The soft spectrum and derived high luminosity $\left(\sim 2 \times 10^{37} \mathrm{erg} \mathrm{s}^{-1}\right)$ imply that its X-ray emission originates from the central star rather than from the surrounding hot shocked gas. After SMP LMC 29 (Kahabka et al. 2008), this is the second known PN in the LMC to emit soft X-rays. Two such systems are also known in the SMC (SMP SMC 22 and SMP SMC 25; Mereghetti et al. 2010), and from a simple estimate based on the mass ratio of the two galaxies, one would expect many more to be detected in the LMC. This deficit could be caused partly by the lower coverage of the LMC with X-ray observations, but also by the stronger diffuse soft X-ray emission in the LMC, which reduces the sensitivity for the detection of faint point sources with soft X-ray spectra.

\subsection{XMMUJ051854.8-695601 and XMMU J050815.1-691832: Galactic soft intermediate polars}

J0518 and J0508 show very similar spectral properties. Their soft blackbody-like emission can be characterised by a temperature between $70-100 \mathrm{eV}$, and both show a hard tail dominating the spectrum above $2 \mathrm{keV}$, an absorption-corrected X-ray luminosity of a few $10^{35} \mathrm{erg} \mathrm{s}^{-1}$ (assuming LMC distance), and a corresponding size of the emission area of $\sim 100-400 \mathrm{~km}$ in radius. All of the above is reminiscent of the properties of soft IPs, except that the luminosities and emission areas are larger than those measured from soft IPs in our Galaxy (for a comparison, see 
Table 4. Cataclysmic variables with blackbody-like soft X-ray emission and fast spinning WDs.

\begin{tabular}{|c|c|c|c|c|c|c|c|}
\hline Source & Other name & Periods ${ }^{(a)}$ & $\begin{array}{l}\text { Distance }^{(b)} \\
(\mathrm{pc})\end{array}$ & $\begin{array}{l}k T^{(c)} \\
(\mathrm{eV})\end{array}$ & $\begin{array}{c}L_{\mathrm{BB}} \\
\left(\mathrm{erg} \mathrm{s}^{-1}\right)\end{array}$ & $\begin{array}{l}R_{\mathrm{BB}} \\
(\mathrm{km})\end{array}$ & $\begin{array}{c}\text { Fluxes }{ }^{(d)} \\
\left(\mathrm{erg} \mathrm{cm}^{-2} \mathrm{~s}^{-1}\right)\end{array}$ \\
\hline RX J0512.2-3241 & UU Col & $863.5 \mathrm{~s} / 3.45 \mathrm{~h}$ & 2429 & 73 & $2.1 \times 10^{32}$ & 7.6 & $5.1 \times 10^{-13}-3.5 \times 10^{-12}$ \\
\hline RX J0558.0+5353 & V 405 Aur & $545.5 \mathrm{~s} / 4.15 \mathrm{~h}$ & 662 & 64.8 & $2.3 \times 10^{32}$ & 31.8 & $1.0 \times 10^{-11}-2.3 \times 10^{-11}$ \\
\hline 1E $0830.9-2238$ & WX Pyx & $1557.3 \mathrm{~s} / \sim 5.54 \mathrm{~h}$ & 1591 & 82 & $1.8 \times 10^{32}$ & 5.6 & $2.3 \times 10^{-13}-4.9 \times 10^{-13}$ \\
\hline RE $0751+14$ & PQ Gem & $833.7 \mathrm{~s} / 5.19 \mathrm{~h}$ & 750 & 47.6 & $9.0 \times 10^{32}$ & 36.9 & $4.7 \times 10^{-12}-6.2 \times 10^{-11}$ \\
\hline 1RXS J154814.5-452845 & NY Lup & $693.0 \mathrm{~s} / 9.87$ & 1228 & 104 & $7.8 \times 10^{32}$ & 7.2 & $4.7 \times 10^{-12}-1.8 \times 10^{-11}$ \\
\hline
\end{tabular}

Notes. ${ }^{(a)}$ Spin period of the WD and binary period. ${ }^{(b)}$ Distances from Gaia DR2 (Bailer-Jones et al. 2018). ${ }^{(c)}$ Blackbody temperatures and bolometric fluxes to compute the bolometric luminosity, $L_{\mathrm{BB}}$, and the emission radius, $R_{\mathrm{BB}}$, of the blackbody component are taken from Evans \& Hellier (2007). ${ }^{(d)}$ Observed flux range in the $0.2-2 \mathrm{keV}$ band; for details, see Sect. 5.

Table 4). Alternatively, the two sources could be located at distances of around $1 \mathrm{kpc}$, which would make their luminosities and emission radii consistent with the typical values measured from Galactic soft IPs. For this purpose, we simplified the spectral model by fitting only one column density for gas with solar elemental abundances. The inferred best-fit parameters are listed in the lower part of Table 3 and are consistent with those measured for Galactic soft IPs (Table 4).

J0518 has likely been a persistent SSS since at least the 1990s, as the detections in archival ROSAT and Swift data and the recent eROSITA CalPV observations suggest. A variation in the amount of absorption column density is, however, observed between the XMM-Newton and eROSITA observations from 2012 and 2019, respectively, leading to significantly different observed fluxes. J0508 exhibits variability on timescales of hours, as seen in the form of flaring behaviour in the XMMNewton observation. Intermediate polars are known to exhibit flaring behaviour on short timescales, which may arise in the turbulent inner region of the accretion disk or from bright spots on the surface of the WD (Barbera et al. 2017; Hellier 2001). The largest flux variations on long-term timescales, by about a factor of six, were also seen from J0508 (Fig. 6), which could be caused by flaring activity similar to that seen during the XMM-Newton observation (Fig. 5). For comparison, we used the HIgh-energy LIght-curve GeneraTor ${ }^{7}$ to produce $0.2-2 \mathrm{keV}$ light curves from archival ROSAT, XMM-Newton, and Swift observations of the Galactic soft IPs that are listed in Table 4. From the available spectral models, we selected the blackbody with $k T=60 \mathrm{eV}$ and $N_{\mathrm{H}}=1 \times 10^{21} \mathrm{~cm}^{-2}$, and the inferred minimum and maximum fluxes are summarised in Table 4 . The somewhat higher variability of the Galactic soft IPs, as seen in Table 4, is likely due to the larger number of available observations compared to the newly discovered systems.

\subsection{XMMU J044626.6-692011}

The X-ray spectrum and the pulsations of $\sim 497 \mathrm{~s}$ suggest an $\mathrm{mCV}$ nature for $\mathrm{J} 0446$ as well. The coherent pulsations denote the spin period of the WD. Several other SSSs with measured spin periods were discovered in XMM-Newton observations of M31: XMMU J004319.4+411758, a transient source with period of $\sim 865 \mathrm{~s}$ (Osborne et al. 2001), and XMMU J004252.5+411540, with a period of $\sim 218 \mathrm{~s}$ (Trudolyubov \& Priedhorsky 2008). In one case, an SSS with 1110 s pulsations could be identified with nova M31N 2007-12b (Pietsch et al. 2011), strongly suggesting that the nova erupted in an IP system and that residual nuclear surface burning is detected after the ejected envelope became optically thin for

\footnotetext{
7 http://xmmuls.esac.esa.int/upperlimitserver/
}

soft X-rays. The spin period measurement suggests an orbital period of $\geq 1.4 \mathrm{hrs}$ for $\mathrm{J} 0446\left(P_{\text {spin }} \sim 0.1 P_{\text {orb }}\right.$; Norton et al. 2004). However, the location of the source, either in the LMC or our Galaxy, is less clear. Assuming the best-fit spectral parameters, the luminosity and size of the emission region at LMC distance are consistent with a WD with nuclear burning on its surface near the Eddington limit. In this case, J0446 could be similar to 1RXS J050526.3-684628 in the LMC, which exhibits $170 \mathrm{~s}$ pulsations (Vasilopoulos et al. 2020). The soft X-ray emission from both sources could be due to residual nuclear surface burning after a nova eruption. While the evolution of the X-ray luminosity of 1RXS J050526.3-684628 could be followed for about 30 years (the first detections were in ROSAT data), J0446 was not detected during the ROSAT all-sky survey, implying that at least the onset of the super-soft phase - and probably also the nova outburst - of J0446 occurred later than that of 1RXS J050526.3-684628.

On the other hand, the spectral model for J0446 with one absorption component yields an absorption-corrected 0.1$2.4 \mathrm{keV}$ luminosity of $5 \times 10^{33} \mathrm{erg} \mathrm{s}^{-1}$ for a distance of $1 \mathrm{kpc}$. The expected $L_{\mathrm{x}}$ of IPs range from $3 \times 10^{29} \mathrm{erg} \mathrm{s}^{-1}$ to $5 \times$ $10^{33} \mathrm{erg} \mathrm{s}^{-1}$ (Ruiter et al. 2006), and with the above argument, J0446 could be a soft IP located in our Galaxy at a distance of $\leq 1 \mathrm{kpc}$. The measured $N_{\mathrm{H}}$ is higher than the expected total Galactic column density in the direction of the source $\left(N_{\mathrm{H}}=N_{\mathrm{HI}}+2 N_{\mathrm{H}_{2}} \approx 2 \times 10^{21} \mathrm{~cm}^{-2}\right.$; Willingale et al. 2013). This is indicative of obscuration local to the binary system, which is common in IPs. Future deeper observations to firmly establish the presence of the hard tail in the X-ray spectrum can help to ascertain the IP nature of this source or, if instead its absence is confirmed, allow the IP nature to be disregarded.

\subsection{New Galactic magnetic cataclysmic variables}

From our four new sources, which show super-soft X-ray emission, at least two (J0518 and J0508) are most likely located in our own Galaxy. Kahabka \& Haberl (2006) reported the XMMNewton detection of the SSS candidate RX J0059.4-7118 in the direction of the SMC and confirmed its super-soft X-ray spectrum, which is characterised by a blackbody component with a temperature of $\sim 90 \mathrm{eV}$ and an additional hard spectral component. Similarly, the high luminosity of RX J0059.4-7118 of $\sim 4 \times 10^{34} \mathrm{erg} \mathrm{s}^{-1}$, if located at SMC distance, let the authors argue for a Galactic $\mathrm{mCV}$. Given the similarities of RX J0059.4-7118 with J0518 and J0508, we support this conclusion and suggest the object to be a soft IP located in our Galaxy. Another case with super-soft X-ray emission in the direction of the LMC is the double-degenerate candidate 3XMM J051034.6-670353, which exhibits $1418 \mathrm{~s}$ X-ray 
pulsations (Haberl et al. 2017). For an LMC distance, this system exhibits a luminosity of around $5 \times 10^{34} \mathrm{erg} \mathrm{s}^{-1}$ and a radius for the emitting area of $\sim 180 \mathrm{~km}$. The detection of a $23.6 \mathrm{~min}$ periodic modulation in the optical and identification of the optical counterpart confirms a location of the binary system in our Galaxy (Ramsay et al. 2018).

J0518 and J0508, together with RX J0059.4-7118 and 3XMM J051034.6-670353, make a significant contribution to the known population of such systems in our Galaxy (Haberl et al. 2017; Anzolin et al. 2008; Evans \& Hellier 2007). The distance to J0446 is less clear, but it could be another IP system in our Galaxy. Evans \& Hellier (2007) argue that whether IPs show a soft emission component mainly depends on the viewing geometry. Accretion curtains hide the soft emission from the surface of the WD in the majority of IPs. In addition to the obscuration intrinsic to binary systems, the interstellar absorption along the line of sight plays an important role in the detection of the soft emission. A higher density of known sources in the direction of the Magellanic Clouds can likely be explained by the relatively low Galactic column density and the large number of existing X-ray observations sensitive to low energies.

Acknowledgements. We thank the referee for useful comments and suggestions which helped to improve the manuscript. This work used observations obtained with XMM-Newton, an ESA science mission with instruments and contributions directly funded by ESA Member States and NASA. The XMM-Newton project is supported by the DLR and the Max Planck Society. This research has made use of the VizieR catalogue access tool, CDS, Strasbourg, France. The original description of the VizieR service was published in A\&AS 143, 23. This work made use of data supplied by the UK Swift Science Data Centre at the University of Leicester. The OGLE project has received funding from the National Science Centre, Poland, grant MAESTRO 2014/14/A/ST9/00121 to AU. This work used data from eROSITA, the soft X-ray instrument on board $S R G$, a joint RussianGerman science mission supported by the Russian Space Agency (Roskosmos), in the interests of the Russian Academy of Sciences represented by its Space Research Institute (IKI), and the Deutsches Zentrum für Luft- und Raumfahrt (DLR). The SRG spacecraft was built by Lavochkin Association (NPOL) and its subcontractors, and is operated by NPOL with support from the Max Planck Institute for Extraterrestrial Physics (MPE). The development and construction of the eROSITA X-ray instrument was led by MPE, with contributions from the Dr. Karl Remeis Observatory Bamberg \& ECAP (FAU Erlangen-Nürnberg), the University of Hamburg Observatory, the Leibniz Institute for Astrophysics Potsdam (AIP), and the Institute for Astronomy and Astrophysics of the University of Tübingen, with the support of DLR and the Max Planck Society. The Argelander Institute for Astronomy of the University of Bonn and the Ludwig Maximilians Universität Munich also participated in the science preparation for eROSITA. The eROSITA data shown here were processed using the eSASS software system developed by the German eROSITA consortium.

\section{References}

Anzolin, G., de Martino, D., Bonnet-Bidaud, J.-M., et al. 2008, A\&A, 489, 1243

Arnaud, K. A. 1996, in Astronomical Data Analysis Software and Systems V, ASP Conf. Ser., 101, 17

Bailer-Jones, C. A. L., Rybizki, J., Fouesneau, M., Mantelet, G., \& Andrae, R. 2018, AJ, 156, 58

Barbera, E., Orlando, S., \& Peres, G. 2017, A\&A, 600, A105

Boller, T., Freyberg, M. J., Trümper, J., et al. 2016, A\&A, 588, A103

Brunner, H., Liu, T., Lamer, G., et al. 2021, A\&A, submitted, [arXiv:2106.14517]
Burwitz, V., Reinsch, K., Beuermann, K., \& Thomas, H.-C. 1996, A\&A, 310, L25

Carpano, S., Haberl, F., Maitra, C., et al. 2021, A\&A, submitted, [arXiv:2106.14536]

Cropper, M. 1990, Space Sci. Rev., 54, 195

Cropper, M., Harrop-Allin, M. K., Mason, K. O., et al. 1998, MNRAS, 293, L57

Dickey, J. M., \& Lockman, F. J. 1990, ARA\&A, 28, 215

Esposito, P., Israel, G. L., Dall'Osso, S., \& Covino, S. 2014, A\&A, 561, A117

Evans, P. A., \& Hellier, C. 2007, ApJ, 663, 1277

Evans, P. A., Beardmore, A. P., Page, K. L., et al. 2007, A\&A, 469, 379

Evans, P. A., Beardmore, A. P., Page, K. L., et al. 2009, MNRAS, 397, 1177

Greiner, J. 1996, in Supersoft X-Ray Sources, ed. J. Greiner (Berlin Springer

Verlag), Lect. Notes Phys., 472, 299

Haberl, F., \& Motch, C. 1995, A\&A, 297, L37

Haberl, F., Sturm, R., Ballet, J., et al. 2012, A\&A, 545, A128

Haberl, F., Israel, G. L., Rodriguez Castillo, G. A., et al. 2017, A\&A, 598, A69

Haberl, F., Maitra, C., Carpano, S., et al. 2021, A\&A, in press, https://doi . org/10.1051/0004-6361/202141878

Hellier, C. 2001, Cataclysmic Variable Stars (Springer)

Kahabka, P., \& van den Heuvel, E. P. J. 1997, ARA\&A, 35, 69

Kahabka, P., \& Haberl, F. 2006, A\&A, 452, 431

Kahabka, P., Haberl, F., Pakull, M., et al. 2008, A\&A, 482, 237

Kuulkers, E., Norton, A., Schwope, A., \& Warner, B. 2006, in X-rays from cataclysmic variables, eds. W. H. G. Lewin, \& M. van der Klis, 421

Lomb, N. R. 1976, Ap\&SS, 39, 447

Long, K. S., Helfand, D. J., \& Grabelsky, D. A. 1981, ApJ, 248, 925

Maggi, P., Haberl, F., Kavanagh, P. J., et al. 2016, A\&A, 585, A162

Maitra, C., Haberl, F., Ivanov, V. D., Cioni, M.-R. L., \& van Loon, J. T. 2019 A\&A, 622, A29

Maitra, C., Haberl, F., Sasaki, M., et al. 2021, A\&A, in press, https://doi . org/10.1051/0004-6361/202141104

Mereghetti, S., Krachmalnicoff, N., La Palombara, N., et al. 2010, A\&A, 519, A42

Morgan, D. H. 1984, MNRAS, 208, 633

Mukai, K. 2017, PASP, 129, 062001

Norton, A. J., Wynn, G. A., \& Somerscales, R. V. 2004, ApJ, 614, 349

Osborne, J. P., Borozdin, K. N., Trudolyubov, S. P., et al. 2001, A\&A, 378, 800

Pietrzyński, G., Graczyk, D., Gieren, W., et al. 2013, Nature, 495, 76

Pietsch, W., Henze, M., Haberl, F., et al. 2011, A\&A, 531, A22

Predehl, P., Andritschke, R., Arefiev, V., et al. 2021, A\&A, 647, A1

Ramsay, G., Marsh, T. R., Kupfer, T., et al. 2018, A\&A, 617, A88

Reid, W. A., \& Parker, Q. A. 2006, MNRAS, 373, 521

Rolleston, W. R. J., Trundle, C., \& Dufton, P. L. 2002, A\&A, 396, 53

Roming, P. W. A., Kennedy, T. E., Mason, K. O., et al. 2005, Space Sci. Rev., 120,95

Ruiter, A. J., Belczynski, K., \& Harrison, T. E. 2006, ApJ, 640, L167

Sasaki, M., Haberl, F., \& Pietsch, W. 2000, A\&AS, 143, 391

Scargle, J. D. 1982, ApJ, 263, 835

Schlafly, E. F., \& Finkbeiner, D. P. 2011, ApJ, 737, 103

Stanghellini, L., Blades, J. C., Osmer, S. J., Barlow, M. J., \& Liu, X. W. 1999, ApJ, 510, 687

Stanghellini, L., García-Lario, P., García-Hernández, D. A., et al. 2007, ApJ, 671, 1669

Strüder, L., Briel, U., Dennerl, K., et al. 2001, A\&A, 365, L18

Sturm, R., Haberl, F., Pietsch, W., et al. 2013, A\&A, 558, A3

Trudolyubov, S. P., \& Priedhorsky, W. C. 2008, ApJ, 676, 1218

Turner, M. J. L., Abbey, A., Arnaud, M., et al. 2001, A\&A, 365, L27

Udalski, A., Szymanski, M., Kaluzny, J., Kubiak, M., \& Mateo, M. 1992, Acta Astron., 42, 253

Udalski, A., Szymański, M. K., \& Szymański, G. 2015, Acta Astron., 65, 1

van den Heuvel, E. P. J., Bhattacharya, D., Nomoto, K., \& Rappaport, S. A. 1992, A\&A, 262, 97

Vasilopoulos, G., Koliopanos, F., Woods, T. E., et al. 2020, MNRAS, 499, 2007

Willingale, R., Starling, R. L. C., Beardmore, A. P., Tanvir, N. R., \& O'Brien, P. T. 2013, MNRAS, 431, 394

Wilms, J., Allen, A., \& McCray, R. 2000, ApJ, 542, 914

Wolf, W. M., Bildsten, L., Brooks, J., \& Paxton, B. 2013, ApJ, 777, 136

Zaritsky, D., Harris, J., Thompson, I. B., \& Grebel, E. K. 2004, AJ, 128, 1606 